\title{
On the anisotropy of seismic waves in the Carpathian region
}

\author{
Eugenia Lyskova ${ }^{1}$ and Konstantin Sannikov \\ St. Petersburg State University, St. Petersburg, Russia
}

\begin{abstract}
The anisotropy of seismic waves in the continental regions still belongs to the category of controversial issues, since its estimates in different areas show a different sign of the anisotropy coefficient. In contrast to studies of oceanic regions, where $\mathrm{SH}$ velocities always prevail over SV velocities, in the continental regions the relations between the velocities are very different. The explanation for this, first of all, is the difference in structure. The structure of the crust and upper mantle under the oceans is much more homogeneous in comparison with the structure of the continental regions. There are several approaches to the estimation of anisotropy. The most traditional method is to use the maximum amount of data separately for Love and Rayleigh waves to study the lateral distributions of SH- and SV-wave velocity, despite the fact that the density of the coverage by paths, and, consequently, the regions of best resolution can be of different shapes and sizes. It was decided to use this method as the first approximation in creating an anisotropic portrait of the Carpathian region. The Carpathian region was chosen as the object of study, since it contains interesting contrasting features: (1) the Pannonian Basin, which is characterized by a thin crust, a thinned lithosphere, and anomalously high values of the heat flux; (2) the Tornquist-Teisseyre zone, which is parallel to the strike of the Eastern Carpathians, and represents the contact zone of the Precambrian lithosphere of the EEP and the relatively young lithosphere of Western Europe. (3) The third feature is the Vrancea zone, one of the most active seismic zones in Europe. It is located in the junction of young tectonic structures: the Southern and Eastern Carpathians, the Transylvanian Depression and the Pre-Carpathian Depression. The results of the study confirm that the Tornquist-Teisseyre Zone divides the structures of the ancient East European Platform and orogenic zones of Western Europe: the upper mantle throughout EEP is characterized by high velocities, whereas velocities throughout WE are markedly lower. Low velocity anomalies prevail under Pannonian Basin which is characterized by anomalously high heat flow values. The distribution of the anisotropy coefficient demonstrates an extended layer of low values of the anisotropy coefficient at depths of $150-200 \mathrm{~km}$. Above this layer, velocity distributions reveal the block structure of the lithosphere. The earthquake sources in the Vrancea zone fall into the transition zone from the highvelocity mantle under the EEP to the low-velocity mantle under the WE. Earthquakes do not occur below the revealed asthenospheric layer.
\end{abstract}

1 Corresponding author: e.lyskova@spbu.ru 


\section{Introduction}

For a long time, studies of the Earth's structure were carried out under the assumption that the Earth is isotropic, while seismic anisotropy was considered to be a second-order effect. However, in the 60s of the 20th century it was shown that most parts of the Earth exhibit anisotropic properties and many effects that were inexplicable within the isotropic model were explained by using the models with anisotropy. Numerous studies show that the Earth's crust and upper mantle exhibit anisotropic properties.

The anisotropy of the upper mantle is evidenced by studies of the structure of the Earth based on the analysis of surface and body waves. The surface wave's data can reveal both radial and azimuthal anisotropy of seismic wave velocities. Radial anisotropy of the upper part of the Earth results in impossibility to satisfy dispersion curves of Rayleigh and Love waves by a unique S-wave velocity-depth curve. Rayleigh wave dispersion curves correspond to SV-wave velocities, whereas Love wave curves are responsible for SH velocities. Analysis of Rayleigh and Love wave dispersion curves inverting to S-wave velocity-depth curves along oceanic paths has shown a predominance of $\mathrm{SH}$ wave velocities over SV wave velocities in the oceanic upper mantle up to $200 \mathrm{~km}$. Since the oceanic crust and upper mantle are almost homogeneous laterally and, on the other hand, 2/3 of the Earth's surface is occupied by the oceans, information about the radial anisotropy of the S-wave velocities in the Earth was included in the PREM model [1]. Anisotropy coefficient was defined as a relative difference between $\mathrm{SH}$ and SV velocities. It reaches the $4.8 \%$ under the Moho boundary, and then it decreases with depth and becomes a zero starting from the depth of $220 \mathrm{~km}$.

The anisotropy of seismic waves in the continental regions still belongs to the category of controversial issues. In contrast to studies of oceanic regions, where $\mathrm{SH}$ velocities always prevail over SV velocities, in the continental regions the relations between the velocities are very different. In [2], the radial anisotropy in Tibet was studied according to Rayleigh and Love waves. Both the excess of the velocity of the SH waves with respect to $\mathrm{SV}$ and the inverse ratio were found, with the anisotropy coefficient varying from $-10 \%$ to $+10 \%$. A similar result for the radial anisotropy of the crust in the Himalayas and Tibet was obtained in [3]. Yanovskaya and Kozhevnikov (2006) used the data on the group velocity dispersion of Rayleigh and Love waves along the paths crossing the Asian continent, and obtained lateral velocity variations of SV and SH waves at different depths. It was found that $\mathrm{SH}$ velocities exceed the velocities of SV up to $6 \%$ at the depths of $100-200 \mathrm{~km}$ in Baikal rift region and in Tibet, but under Siberian Platform the anisotropy was not practically revealed [4].The explanation for this, first of all, is the difference in structure. The structure of the crust and upper mantle under the oceans is much more homogeneous in comparison with the structure of the continental regions.

Estimations of the radial anisotropy in the European region are rather discrepant [5-10]. In particular, in the model proposed in [7] some zones with $\mathrm{V}_{\mathrm{SV}}>\mathrm{V}_{\mathrm{SH}}$ were revealed, whereas in [9] $\mathrm{V}_{\mathrm{SH}}>\mathrm{V}_{\mathrm{SV}}$ was found everywhere.

All approaches to the analysis of radial anisotropy can be conditionally divided into two large groups. The studies from the 1st group are carried out in accordance with the 3D $\rightarrow 2 \mathrm{D}+1 \mathrm{D}$ algorithm. At the first stage, lateral variations of the group velocities of the Rayleigh and Love waves for different periods (2D) are usually obtained from the dispersion curves of surface waves using tomographic methods. Then, in the region of the best resolution, "local dispersion curves" are inverted to the "local S-wave velocity-depth curves" (1D). The dispersion curves of the Rayleigh waves reconstruct the velocity distribution $V_{S V}(\varphi, \lambda, z)$, the dispersion curves of the Love waves - the distribution $V_{S H}(\varphi, \lambda, z)$, and, finally, the three-dimensional distribution of the anisotropy 
coefficient $\alpha(\varphi, \lambda, z)$ can be obtained. This approach was widely used in studies of different regions of the Earth [11-16].

In $[17,18]$, the surface wave tomography was directly applied to anisotropy coefficient for each wave path rather than to dispersion curves separately. In other words, 3D inversion in the form of $3 \mathrm{D} \rightarrow 2 \mathrm{D}+1 \mathrm{D}$, used to determine the velocity distributions, was replaced with the $3 \mathrm{D} \rightarrow 1 \mathrm{D}+2 \mathrm{D}$ scheme to estimate the spatial distribution of the anisotropy coefficient.

The present article is focused to the first way of the seismic waves anisotropy studying in the Carpathian region. We have applied more traditional algorithm $3 \mathrm{D} \rightarrow 2 \mathrm{D}+1 \mathrm{D}$. The Carpathian region was chosen as the object of study, since it contains interesting contrasting features: (1) the Pannonian Basin, which is characterized by a thin crust, a thinned lithosphere, and anomalously high values of the heat flux [19]; (2) the TornquistTeisseyre zone, which is parallel to the strike of the Eastern Carpathians, and represents the contact zone of the Precambrian lithosphere of the East European Platform and the relatively young lithosphere of Western Europe. (3) The third feature is the Vrancea zone, one of the most active seismic zones in Europe. It is located in the junction of young tectonic structures: the Southern and Eastern Carpathians, the Transylvanian Depression and the Pre-Carpathian Depression. The Vrancea zone is characterized by a relatively narrow, almost vertical seismic generating volume of the medium, steeply falling in the north-west direction; location under the thickened continental crust of the Carpathian arc; the mismatch of the position of the hypocenters with a known tectonic suture in the Carpathian orogenic system.

At the next stage of research, we plan to complete the anisotropic portrait of the Carpathian region by estimating the distribution of the anisotropy coefficient using the method proposed by Yanovskaya T.B. $[17,18]$ according to the scheme 3D $\rightarrow 1 \mathrm{D}+2 \mathrm{D}$.

At the final stage, we suppose to use the cleaned dispersion of the Love wave derived from polarization analysis, since it is known that harmonics of different periods can have different paths to the station, and deviations from the geometric azimuth can reach up to 20 degrees.

\section{Methods and Data}

In recent decades, seismic tomography methods have been developed and widely used to study the structure of the Earth. To study the structure of the upper layers of the Earth, which is the most heterogeneous horizontally, the most effective method is using surface wave data - surface wave tomography. Surface-wave tomography allows one to obtain lateral distributions of the group velocities of Love and Rayleigh waves for different periods from the dispersion curves of surface waves. However, the main goal of research is, ultimately, not to determine the lateral variations in the velocities of surface waves, but to find the three-dimensional distribution of transverse velocities in the medium. This information is possible to obtain due to the fact that surface waves penetrate into a depth comparable to the wavelength, and the velocities depend on the wavelength or period. Therefore, the missing information on the structure variations is made up by the fact that the wave velocities for each trace can be used for different periods. Surface waves have a unique property: it lies in the fact that the characteristics of surface waves propagating in media with weak horizontal inhomogeneity are locally the same as in a horizontally homogeneous medium with a vertical distribution of body wave velocities and density under the point under consideration. This circumstance allows us to divide the problem into two stages - two-dimensional tomography of the surface wave velocities for different periods and restoration of the dependence of the velocity on the third coordinate (depth). 
Let us describe the research process in more details. For studying the anisotropy of seismic waves in the upper mantle we should obtain the dispersion curves of both the Rayleigh and Love waves in a fairly wide interval of periods (up to $100 \mathrm{~s}$ ). It is known that group velocities of both Rayleigh waves and Love waves are more sensitive to changes in the $\mathrm{S}$ wave velocity deviations than phase ones. [11, 20]. Therefore, using the timefrequency analysis, we namely obtain the dispersion curves of the group velocities of Love and Rayleigh waves.

To calculate the lateral distributions of the surface wave group velocities for different periods from the obtained dataset of the dispersion curves we should use the method of the surface wave tomography. Most surface wave tomography methods are based on parameterizing the model and finding the model parameters [11, 21, 22]. The study area in these methods is divided into blocks and the average values of the unknown function in each block are estimated. Such approach is efficient where there is a very large amount of seismic paths and the seismic paths are uniformly distributed in azimuths. In the present study, a different tomography method developed by Ditmar and Yanovskaya is used. It is based on smoothness of the lateral velocity variations [23]. This approach has been used more extensively [12-16] as it does not impose restrictions on the quantity and quality of the input data: in the areas where the number of data is insufficient, the resolution is low.

The next step of the tomographic reconstruction is solving the one dimensional (1D) inverse problem, i.e., reconstructing the vertical sections of the velocity at the grid points from the dispersion curves of group velocities. The sought velocity sections are represented in the form of three layers of constant velocity approximating the crust and piecewise linear velocity variations in the underlying mantle. For the numerical calculations of the group velocity, the linear velocity variation is replaced by a set of a large number of homogeneous layers $[13,20]$.

The scheme of algorithm 3D $\rightarrow$ 2D $+1 \mathrm{D}$ is shown in Fig.1. The lateral distributions of the group velocity of the Love / Rayleigh waves for a set of periods (like a layered cake) are schematically shown in the left panel of the Figure 1 (2D). The method developed by Ditmar and Yanovskaya [23] allows one to estimate the radius of the equivalent smoothing area and determine the acceptable resolution region where further the local dispersion curves will be obtained at the grid points. The local dispersion curve of the Rayleigh group velocity for a point with coordinates ( $\varphi, \lambda$ ), as an example, is drawn in the center of the figure. The reconstructed SV-velocity section (1D) is illustrated on the right.

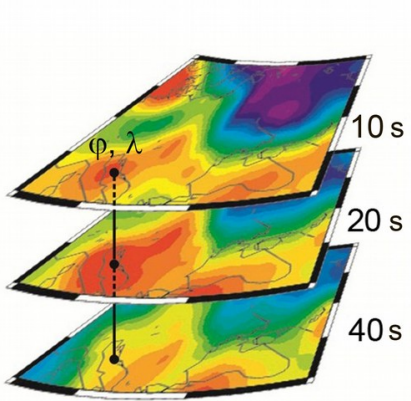

2D

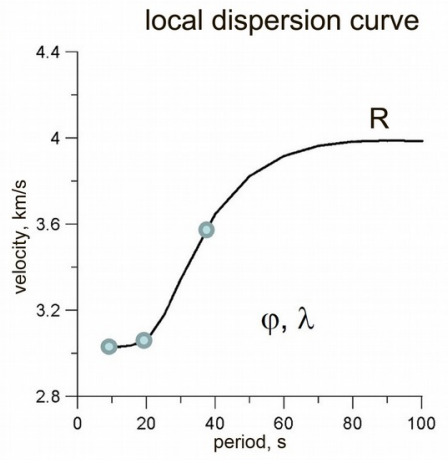

Fig. 1. The scheme of algorithm $3 D \rightarrow 2 D+1 D$. Left panel: the lateral distributions of the group velocity of the Love / Rayleigh waves for a set of periods (2D). Central panel: local dispersion 
curve of the Rayleigh group velocity (for example) for a point with coordinates $(\varphi, \lambda)$. Right panel: reconstructed SV-velocity section (1D).

The dispersion of the Love and Rayleigh waves was determined from the earthquake records. We selected the LH records from the IRIS and GEOFON data centers and determined with confidence the dispersion of both Love and Rayleigh waves along the paths crossing the European continent (Fig. 2). The dispersion curves of the Rayleigh wave velocities are determined from the vertical component of the displacements. To obtain the dispersion curves of the Love waves, the transversal component is used. T-component is determined by transforming the $\mathrm{N}$ and $\mathrm{E}$ components by the angle corresponding to the geometric azimuth. It was also important to exclude parts of records in the vicinity of the Airy phase, where the group velocity has a minimum. Otherwise, you can get false extremes. Finally, another difficulty is the influence of the source mechanism on the recording of the surface wave. Thus, the strike-slip events along sublatitudinal faults in the Turkey-Iran region demonstrated at many stations in Europe a strong Love wave, while the Rayleigh wave amplitude was at the noise level.

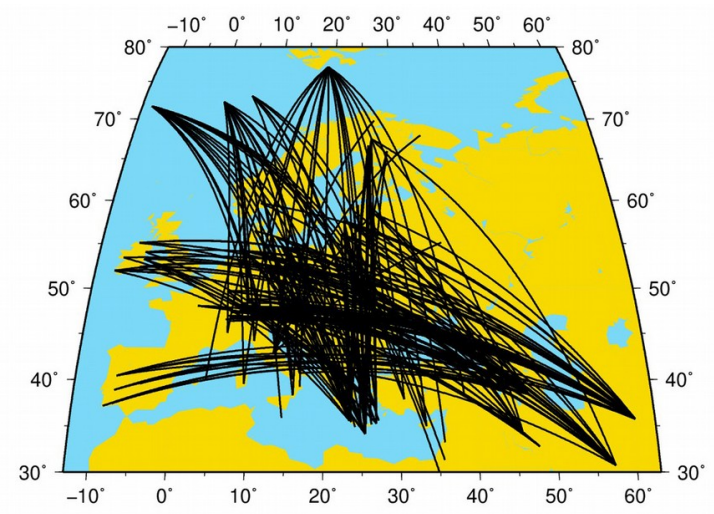

Fig. 2. A map with the paths along which the dispersion curves of the Love and Rayleigh waves were obtained.

As seen from Fig. 2 the densest coverage by seismic paths occurs in the central part of Western Europe, including the Carpathian region. Correspondingly, the highest resolution of the data should also be observed in this area. By coarsely estimating the resolution of the data by the radius $\mathrm{R}$ of the equivalent averaging area [23], one can see that, indeed, the highest resolution is achieved in 2 areas, where $\mathrm{R}$ is about $450 \mathrm{~km}$ (Fig. 3). These areas are marked by red color. We have limited the study area so that the radius does not exceed $600 \mathrm{~km}$.

Therefore, the further stage of research - constructing the local dispersion curves by $2 \mathrm{D}$ tomography and estimating the velocity distribution of $\mathrm{S}$ waves in the upper mantle was conducted in the area of the acceptable resolution, which is delineated in Fig. 4 by black line.

As seen from Fig. 4, the area includes structural features of the Carpathian Pannonian system. It is known that the Pannonian Basin is characterized by a thin crust, a thinned lithosphere, and anomalously high values of the heat flux [19]. The TornquistTeisseyre zone (TTZ) extends in parallel to the strike of the Eastern Carpathians and represents the contact zone of the Precambrian lithosphere of the East European Platform and the relatively young lithosphere of Western Europe. The Vrancea zone is one of the most active seismic zones in Europe. It is located in the junction of young tectonic structures: the Southern and Eastern Carpathians, the Transylvanian Depression and the 
Pre-Carpathian Depression. The Vrancea zone is characterized by a relatively narrow, almost vertical seismic generating volume of the medium, steeply falling in the north-west direction; location under the thickened continental crust of the Carpathian arc; the mismatch of the position of the hypocenters with a known tectonic suture in the Carpathian orogenic system.

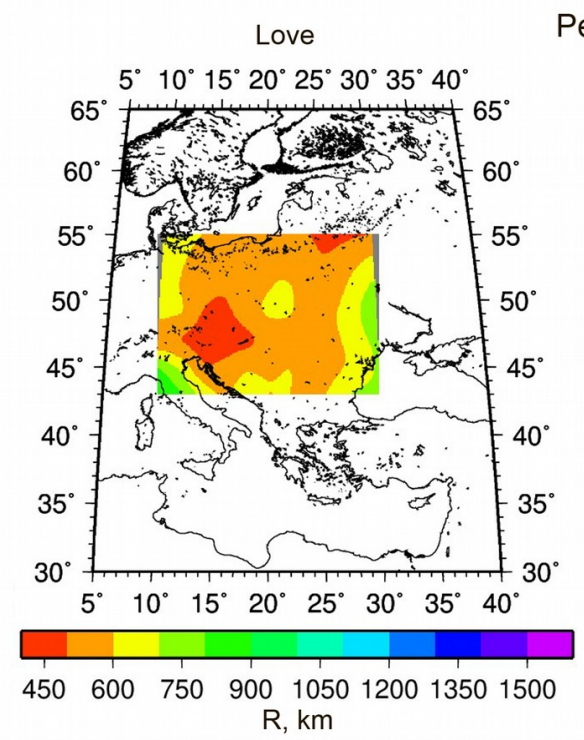

Period $60 \mathrm{~s}$

Rayleigh

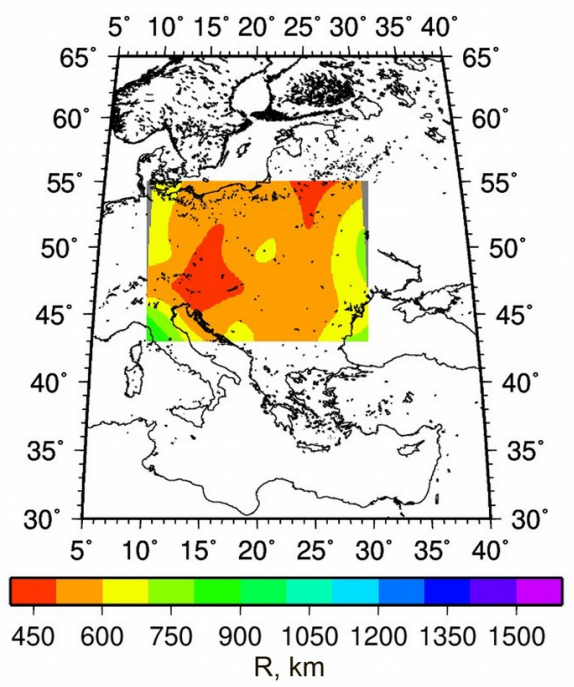

Fig. 3. Distribution of the radius $\mathrm{R}$ of the effective smoothing area.

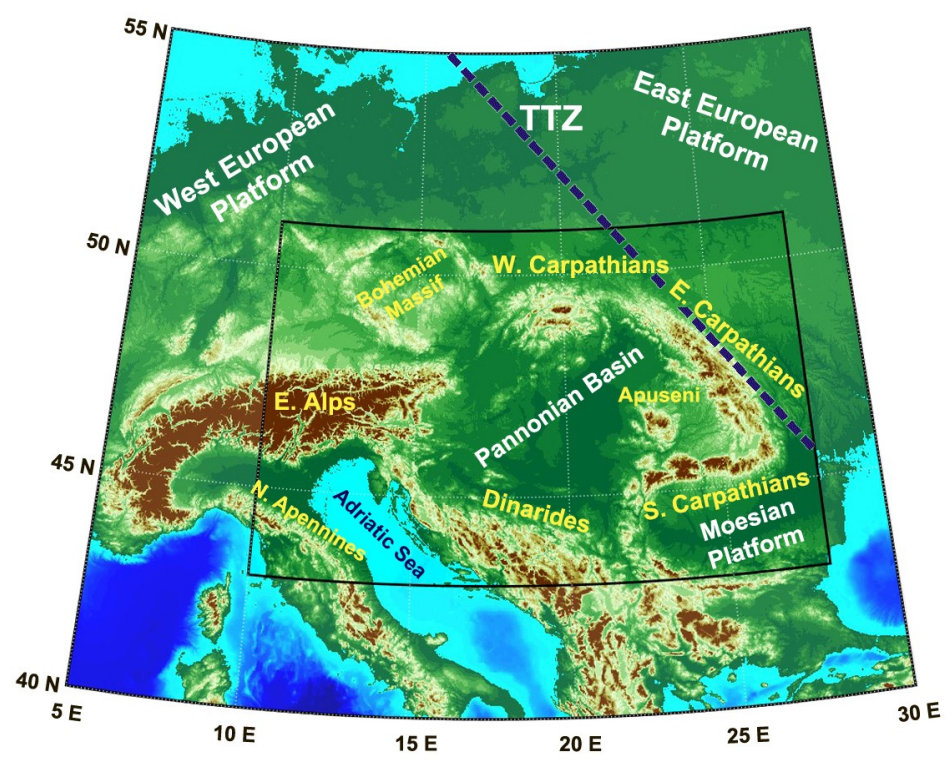

Fig. 4. Topographic map of the Carpathian-Pannonian system with surface structural features. Area of the acceptable resolution is delineated by black line. Tornquist-Teisseyre Zone (TTZ) is marked by blue dashed line. 


\section{Results}

As you can see from Fig.2, the amount of data is moderate. Therefore, the result of tomography should be rather smooth, and small heterogeneities should not be resolved. The area of a satisfactory resolution is contoured by a line in Fig. 4. In this area we calculated the lateral variations of Love and Rayleigh wave group velocity for the periods $10-100 \mathrm{~s}$ by the 2D tomography method based on smoothness of the lateral velocity variations [23]. The calculated lateral variations of Love and Rayleigh wave group velocities allowed us to reveal some preliminary features of large-scale mantle heterogeneities. To determine the depths of the velocity heterogeneities the local dispersion curves were constructed on the basis of the lateral group velocity distributions at grid $1 \times 1$ 。 within the area under study. Then, the local dispersion curves were inverted to the velocity sections using the conjugate gradient method [20]. The figure 5 shows the local dispersion curves of Love and Rayleigh and inverted local velocity sections at the point $\phi=46^{\circ} \mathrm{N}, \lambda=$ $20^{\circ} \mathrm{E}$ in the Pannonian Basin.

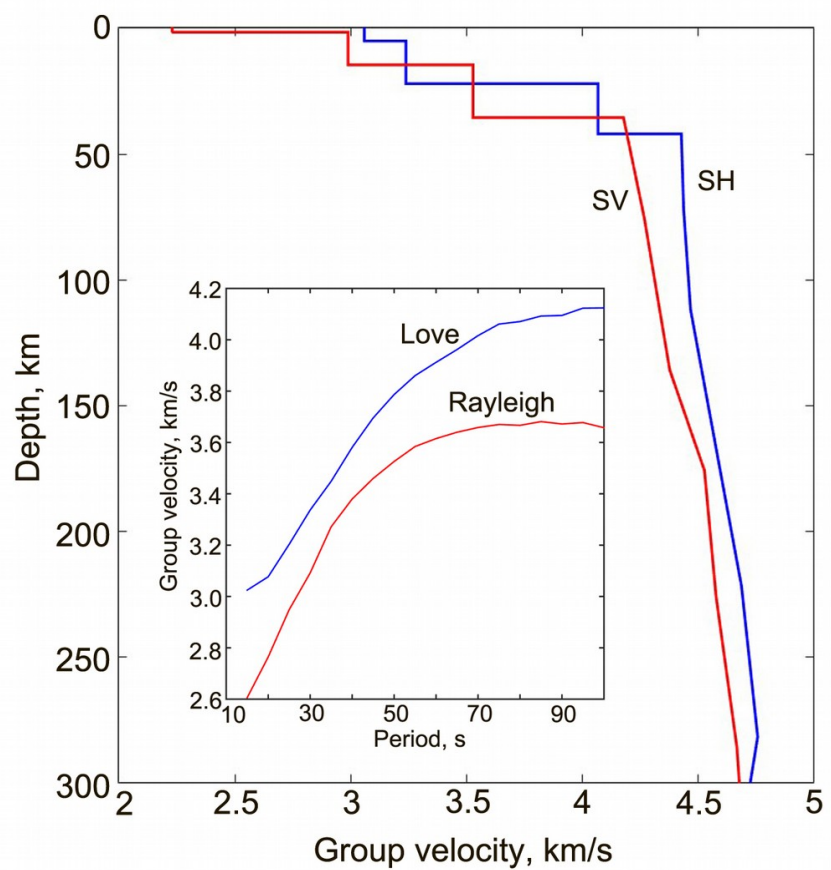

Fig. 5. An example of inversion of a group velocity dispersion curves to local velocity sections at the point $\phi=46^{\circ} \mathrm{N}, \lambda=20^{\circ} \mathrm{E}$ in the Pannonian Basin.

Variations of SV- and SH-wave velocity and anisotropy distributions at depths 75 and $150 \mathrm{~km}$ in the mantle are shown in Fig. 6. As a measure of anisotropy, we take the ratio $\alpha=\left(V_{S H}^{2}-V_{S V}^{2}\right) / V_{S V}^{2}$. The velocity maps clearly trace the Tornquist-Teisseyre zone, which appears here as the line separating the ancient East European Platform (EEP) from 

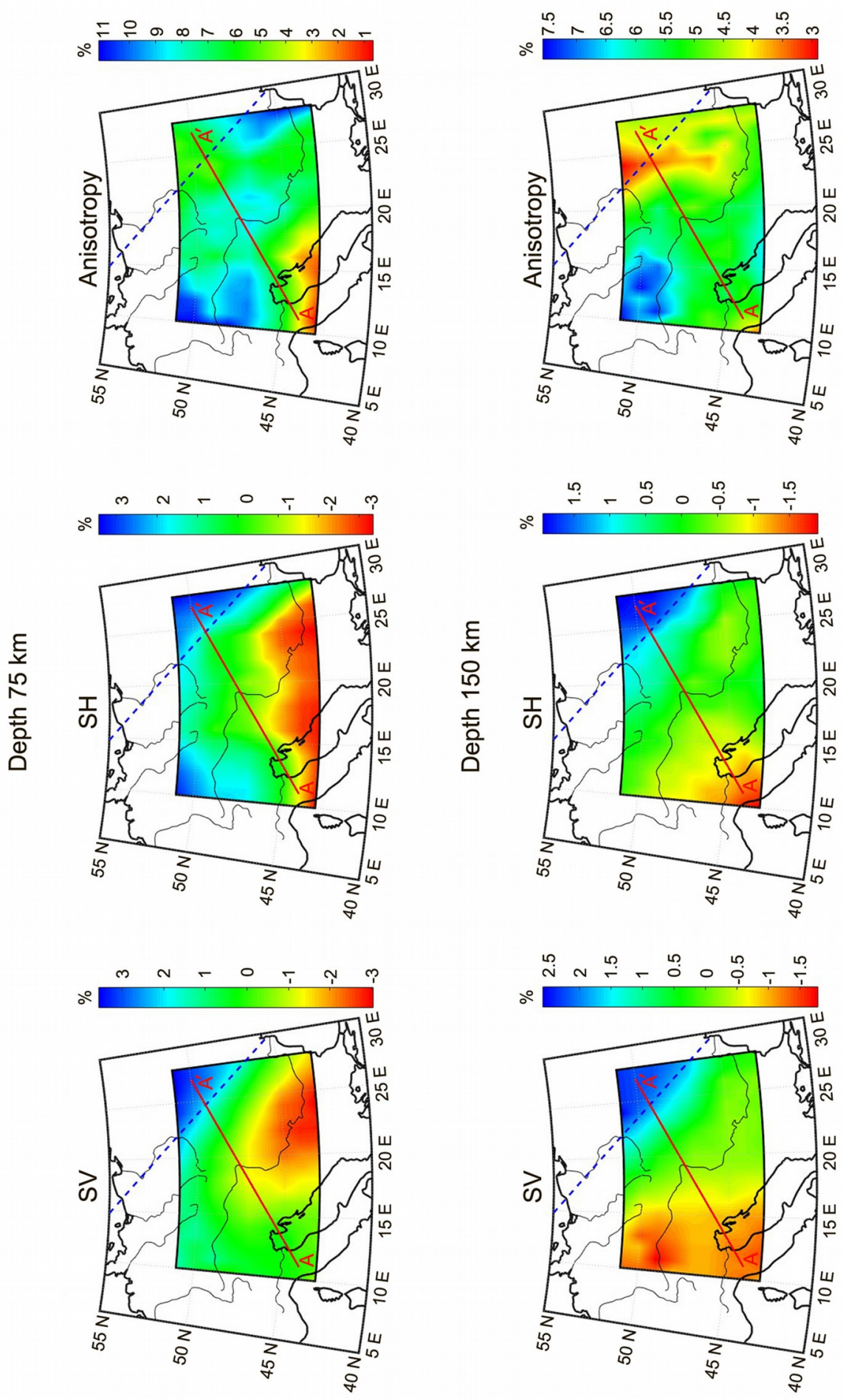

Fig. 6. Variations of SV- and SH-wave velocities and anisotropy coefficient distributions at depths 75 and $150 \mathrm{~km}$ in the mantle. 
the young structures of Western Europe. The Tornquist-Teisseyre Line demarcates different structures at depth ranging from 75 to $150 \mathrm{~km}$. Below in the mantle, velocity differences are smoothed out. For a depth of $75 \mathrm{~km}$, we can see high velocities under EEP and low velocities in Western Europe. The lowest velocities are revealed in the Pannonian Basin. As for the anisotropy, its values are positive for the entire region and for all depths, i.e. the velocities of the SH-wave is greater than the velocities of the SV-wave. Higher values of anisotropy correspond to the area west of the Bohemian Massif, as well as the junction zone of the Eastern and Southern Carpathians. Low values of anisotropy are typical for the region of the Northern Apennines and the central part of the Adriatic.

For a depth of $150 \mathrm{~km}$, we see that the TTZ line still separates the higher-velocity lithosphere of the East European Platform from the slower one corresponding to the West European Platform. At the same time, the area of the lowest velocities shifts from the Pannonian Basin to the west, capturing the north of the Apennine Peninsula, the Adriatic, the Alps and the area under the Bohemian Massif. High values of anisotropy are still characteristic of the region west of the Bohemian Massif. Low values of anisotropy are concentrated along a linear submeridional structure along the 25th degree east longitude.

To reveal the structural features with depth, a section was built along the AA' profile (Fig.7). Profile AA' begins in the Northern Apennines, passes through the northern part of
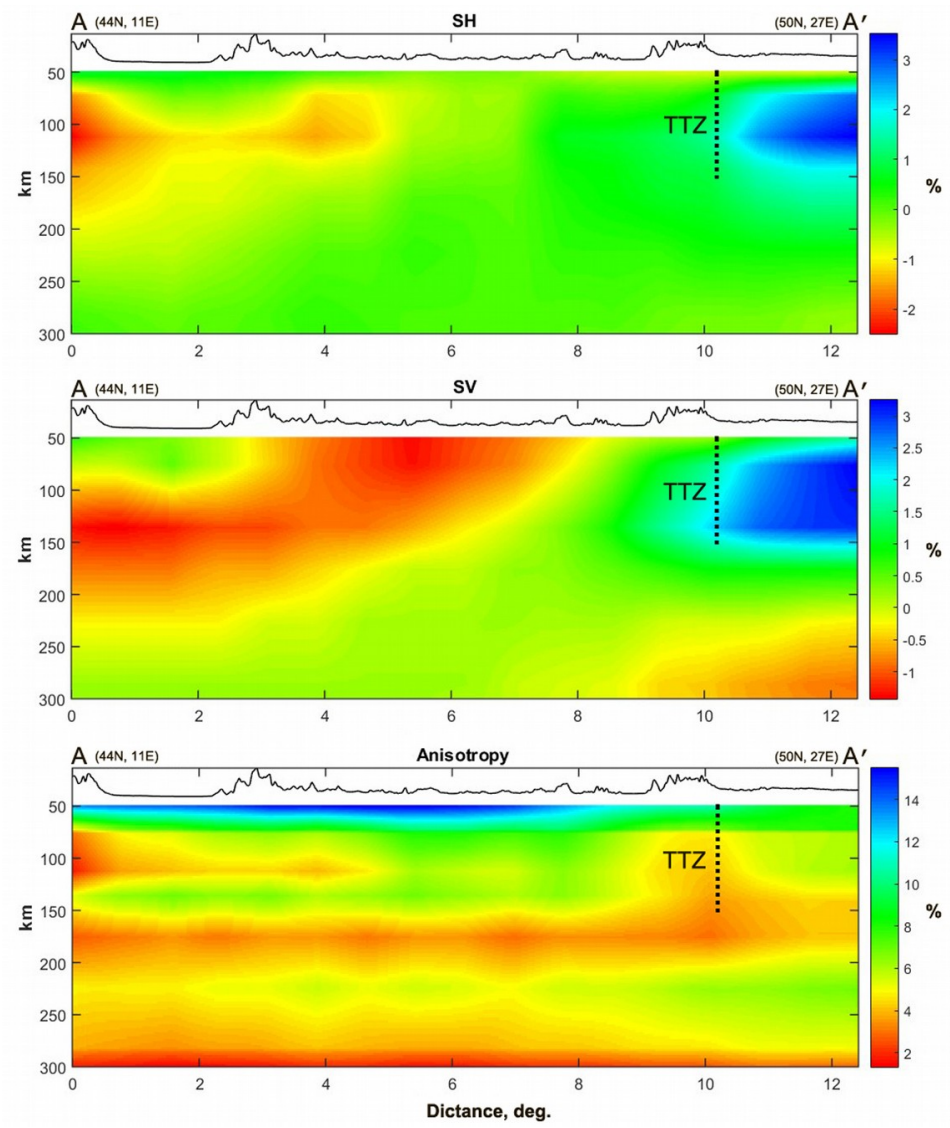

Fig. 7. The vertical distributions of SH- and SV-wave velocities and of anisotropy coefficient along the profile AA' shown in Fig. 6. 
the Adriatic Sea and then follows through the northern Dinarides and the Pannonian Basin, and ends in the West of the East European Plate. The high-velocity EEP lithosphere located to the east of the Tornquist-Teisseyre Zone is clearly traced on the velocity sections for both $\mathrm{SH}-$ and SV-wave velocities. Its thickness is about $70 \mathrm{~km}$ and it is located down to a depth of $150 \mathrm{~km}$. On the cross-section for the SV-wave, the region of lower velocities under the Pannonian Basin is more clearly distinguished. This area, at depths of over $100 \mathrm{~km}$, goes under the Adriatic Sea and the Apennine Peninsula. Anisotropy is characterized by a lateral distribution, with high values in the subcrustal lithosphere of the Pannonian Basin and low values at a depth of about $170 \mathrm{~km}$. The vertical structure with low values of anisotropy is traced near to TTZ area, which, as can be seen in Fig. 6, has a submeredional strike.

To study the structural features in the vicinity Vrancea zone, two intersecting profiles BB' and CC' were built (Fig. 8). They show the hypocenters of earthquakes with a magnitude of 5.0 and higher.
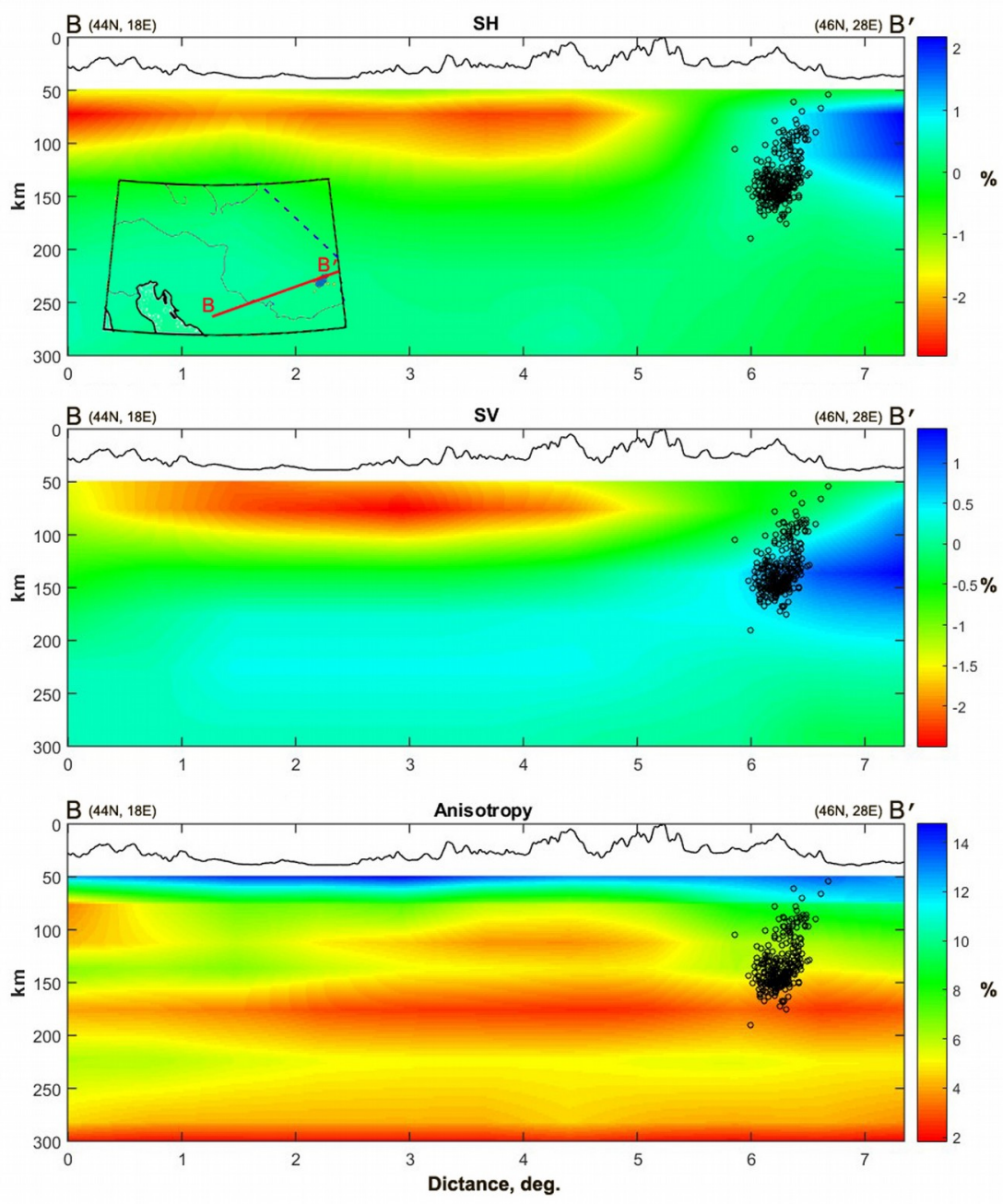

Fig. 8. The vertical distributions of SH- and SV-wave velocities and of anisotropy coefficient along the profile BB'. The position of the profile is shown in the inset at the top of the figure. The hypocenters of earthquakes with a magnitude of 5.0 and higher are shown by circles. 
The BB' profile begins in the Dinarides region, passes through the Pannonian Basin and further through the South Carpathians and the Vrancea zone. The characteristic feature of the profile, as before, is the high-velocity lithosphere of the EEP, reaching depths of 150 $\mathrm{km}$, and the low-velocity lithosphere, located under the Pannonian Basin and the Dinarides. SV waves have higher velocities under the Southern Carpathians at depths greater than 200 $\mathrm{km}$. The earthquakes foci are confined to the edge of the high-velocity lithosphere of the EEP. The anisotropy is characterized by higher values in the subcrustal lithosphere and lower values at a depth of $170 \mathrm{~km}$. Also, low anisotropy values are typical for the area at a depth of $100 \mathrm{~km}$, located under the South Carpathians and Dinarides.

The CC' profile begins in the Pannonian Basin and passes through Transylvania and the Vrancea zone. We see the high velocity lithosphere of the EEP east of the Carpathians and the low velocity lithosphere below the Pannonian Basin. Anisotropy is characterized by a lateral distribution with high values in the subcrustal lithosphere and low values at a depth of about $170 \mathrm{~km}$.
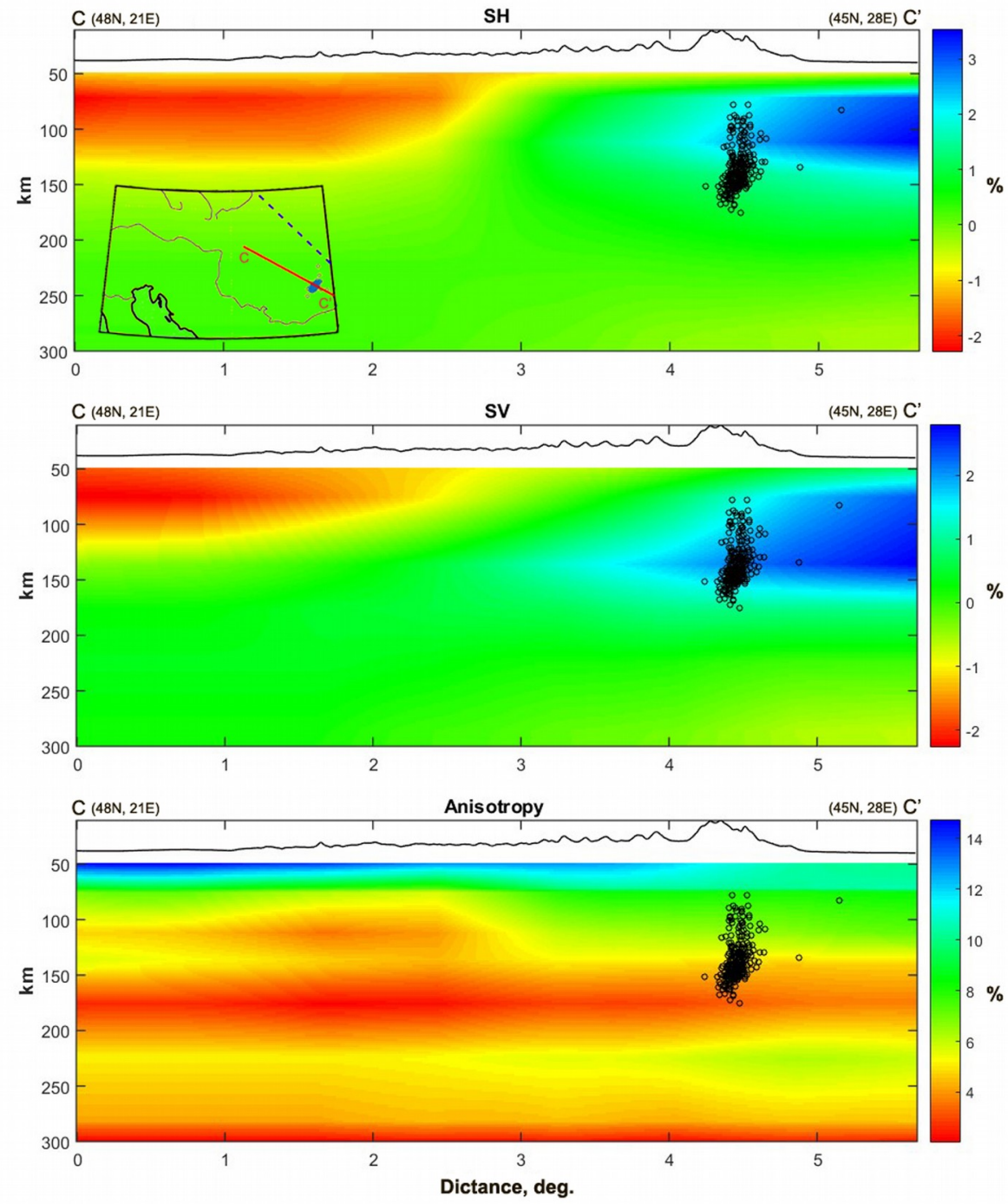

Fig. 9. The vertical distributions of SH- and SV-wave velocities and of anisotropy coefficient along the profile CC'. 


\section{Conclusion}

In the present study, using the method of surface-wave tomography [23] it was possible to determine the three-dimensional distribution of shear wave velocities in the upper mantle to a depth of $300 \mathrm{~km}$ in the area of the complex tectonic structure of the Carpathian region, where heterogeneous structures join together.

Although the amount of data is moderate and the method for constructing the locally averaged dispersion curves applied in this study lead to the smoothing of the resulting velocity sections, the pattern of the velocity distributions revealed in the upper mantle of the Carpathian region well agrees with the known tectonic structures and velocity features obtained by other researchers.

The results obtained confirm that the Tornquist-Teisseyre Zone divides the structures of the ancient East European Platform and orogenic zones of Western Europe: the upper mantle throughout EEP is characterized by high velocities, whereas velocities throughout WE are markedly lower [16].

According to the research results $[24,25]$, one should expect that in the rear of the Eastern Carpathians, low velocities should prevail in comparison with higher velocities in the north east of the arc. This velocity feature is clearly visible from the consideration of lateral velocity distributions in Fig. 6 and vertical distributions of velocities along the AA' and CC' profiles in Fig. 7 and Fig. 9. Low velocity anomalies prevail under Pannonian Basin and Transylvania. The Pannonian Basin, according to [19], is characterized by anomalously high heat flow values.

The distribution of the anisotropy coefficient shown on the vertical sections along the AA', BB' and CC' profiles (Fig. 7, 8, 9) demonstrates an extended layer of low values of the anisotropy coefficient at depths $150-200 \mathrm{~km}$. Above this layer, velocity distributions reveal the block structure of the lithosphere. This circumstance allows us to conclude that this layer is close in its properties to the asthenosphere - for the asthenosphere the difference in the values of SH- and SV-wave velocities should decrease, and hence the anisotropy coefficient will also decrease.

As noted above, earthquake sources in the Vrancea zone fall into the transition zone from the high-velocity mantle under the EEP to the low-velocity mantle under the WE. From Fig. 8 and 9 it follows that below the layer at a depth of 150-200 km, which we consider as asthenospheric, earthquakes do not occur.

The data used in this research were downloaded from the IRIS (https://www.iris.edu/hq) and GEOFON (https://geofon.gfz-potsdam.de) data centres.

We dedicate this paper to the memory of the late Professor Tatiana Yanovskaya, whose contribution to the development of seismic tomography methods and the study of anisotropy was significant.

\section{References}

1. A.M. Dziewonski, D.L. Anderson, Phys. Earth Planet. Inter., 25, 297-356 (1981)

2. Y. Chen, J. Badal, Zh. Zhang, Journal of Asian Earth Science, 36, 289-302 (2009)

3. Zh. Guo, X. Gao, W. Wang, Zh. Yao, Geophys. J. Int., 189, 1169-1182 (2012)

4. T.B. Yanovskaya, V.M. Kozhevnikov, Russ. Geol. Geophys. 47(5), 618-625 (2006)

5. N.M. Shapiro, M.H. Ritzwoller, Geophys. J. Int., 151, 88-105 (2002)

6. B. Kustowski, G. Ekström, A.M. Dziewonski, J. Int., 174, 978-992 (2008) 
7. S.-J. Chang, S. van der Lee, E. Matzel, H. Bedle, Geophys. J. Int., 182(2), 1013-1024 (2010) https://doi.org/10.1111/j.1365-246X.2010.04662.x

8. L. Boschi, B. Fry, G. Ekström, D. Giardini, Surv. Geophys., 30, 463-501 (2009)

9. R. Schivardi, A. Morelli, Geophys. J. Int., 185, 469-484 (2011)

10. J.F. Schaefer, L. Boschi, T.W. Becker, E. Kissling, Geophys. Res. Lett., 38, L23304 (2011). https://doi.org/10.1029/2011GL049687

11. M.H. Ritzwoller, A.L. Levshin, J. Geophys. Res., 103, 4839-4878 (1998)

12. T.B. Yanovskaya, L.M. Antonova, V.M. Kozhevnikov, Phys. Earth and Planet. Int., 122,19-32 (2000)

13. T.B. Yanovskaya, V.M. Kozhevnikov, Phys. Earth and Planet. Int., 138, 263-278 (2003)

14. V.M. Kozhevnikov, A.I. Seredkina, O.A. Solovei, Russ. Geol. Geophys., 55, 12391247 (2014) https://doi.org/10.1016/j.rgg.2014.09.010.

15. A.I. Seredkina, O.A. Solovei, Geodynamics \& Tectonophysics, 9(2), 427-437 (2018). (In Russ.) https://doi.org/10.5800/GT-2018-9-2-0354

16. T. Yanovskaya, T. Koroleva, E. Lyskova, Geophys. J. Int., 205, 1208-1220 (2016) https://doi.org/10.1093/gji/ggw083

17. T.B. Yanovskaya, E.L. Lyskova, T.Yu. Koroleva, Izv. Phys. Solid Earth, 55(2), 195204 (2019)

18. T.B. Yanovskaya, E.L. Lyskova, T.Yu. Koroleva, In: Problems of Geocosmos-2018, Springer Proceedings in Earth and Environmental Sciences (ed. Yanovskaya T.B., Kosterov A.A. et al.). https://doi.org/10.1007/978-3-030-21788-4. (2020)

19. G. Tari, P. Dovenyi, I. Dunkl et al., Geological Society of London. Special Publication, 156, 215-250 (1999)

20. T.B. Yanovskaya, Poverkhnostno-volnovaya tomografiya $\mathrm{v}$ seysmicheskikh issledovaniyakh (Surface Wave Tomography in Seismic Studies) ( Nauka, St.Petersburg, 2015)

21. Y.S. Zhang, T. Tanimto, Geophys. J. Int., 98, 255-269 (1989)

22. M.P. Barmin, M.H. Ritzwoller, A.L. Levshin, Pure Appl.Geophys., 158, 8, 1351-1375 (2001)

23. T.B. Yanovskaya, P.G. Ditmar, Geophys. J. Int., 102(1), 63-72 (1990)

24. B.D.E. Dando, G.W. Stuart, G.A. Houseman, E. Hegedüs, E. Brückl, S. Radovanović, Geophys. J. Int., 186, 11-31 (2011)

25. Y. Ren, G.W. Stuart, G.A. Houseman, B. Dando, C. Ionescu, E. Hegeidus, S. Radivanovich, Y. Shen \& South Carpathian ProjectWorking Group, Earth planet. Sci. Lett., 349-350, 139-152 (2012) 